Case Report

\title{
Canine Staphylococcus argenteus: Case Report from The Netherlands
}

\author{
Eelco F. J. Meijer ${ }^{1, *(\mathbb{D})}$, Anne van Renssen ${ }^{2}$, Ianthe Maat ${ }^{1}$, Linda van der Graaf-van Bloois ${ }^{3} \mathbb{D}$, Birgitta Duim $^{3}$ \\ and Els M. Broens ${ }^{3}$ (D)
}

check for updates

Citation: Meijer, E.F.J.; van Renssen, A.; Maat, I.; van der Graaf-van Bloois, L.; Duim, B.; Broens, E.M. Canine Staphylococcus argenteus: Case Report from The Netherlands. Pathogens 2022, 11, 153. https://doi.org/ $10.3390 /$ pathogens 11020153

Academic Editor: Jilei Zhang

Received: 29 November 2021

Accepted: 24 January 2022

Published: 26 January 2022

Publisher's Note: MDPI stays neutral with regard to jurisdictional claims in published maps and institutional affiliations.

Copyright: (C) 2022 by the authors. Licensee MDPI, Basel, Switzerland. This article is an open access article distributed under the terms and conditions of the Creative Commons Attribution (CC BY) license (https:// creativecommons.org/licenses/by/ $4.0 /)$.
1 Department of Medical Microbiology and Radboud Center for Infectious Diseases, Radboud University Medical Center, 6525 GA Nijmegen, The Netherlands; ianthe.maat@radboudumc.nl

2 Orthopedics Department, Medical Centre for Animals, 1105 AZ Amsterdam, The Netherlands; orthopeed@mcvoordieren.nl

3 Department of Biomolecular Health Sciences, Faculty of Veterinary Medicine, Utrecht University, 3584 CL Utrecht, The Netherlands; L.vanderGraaf@uu.nl (L.v.d.G.-v.B.); B.Duim@uu.nl (B.D.); E.M.Broens@uu.nl (E.M.B.)

* Correspondence: eelco.meijer@radboudumc.nl

\begin{abstract}
Staphylococcus argenteus has been reported worldwide in humans, while reported nonhuman cases are sparse. Its complete epidemiology, alongside its infectivity and pathogenicity in humans and non-humans, remain to be clarified. Here, we describe the first reported canine Staphylococcus argenteus, causing a deep wound infection in a Labrador retriever after orthopedic surgery. The closed genome is reported, with phylogenic and genetic analyses, as well as extensive phenotypic antimicrobial susceptibility testing for human and veterinary antibiotics. No genetic explanation could be found for its interaction with a canine host, underscoring the intrinsic multispecies pathogenicity and potential (anthropo-)zoonotic spread of Staphylococcus argenteus.
\end{abstract}

Keywords: Staphylococcus argenteus; canine; genome sequencing; ST2250

\section{Introduction}

Staphylococcus argenteus has recently been identified as a novel coagulase-positive species within the Staphylococcus aureus complex lacking the carotenoid pigment staphyloxanthin, formerly described as the distinct $S$. aureus lineage clonal complex 75 [1]. Several more clonal complexes have been described to belong to $S$. argenteus since and multiple studies have elaborated on its worldwide spread, including Australia, Africa, Asia, America and Europe [2]. S. aureus and S. argenteus have been implicated in disease in humans and non-humans [2,3], whereas the closely related $S$. schweitzeri has been found primarily in bats and non-human primates and no human infections have been described to date $[4,5]$. Human $S$. argenteus infections are associated with serious morbidity, mortality and nosocomial infection in humans [3,6], and isolates have been shown to harbor most of the virulence genes of $S$. aureus [5]. Non-human described cases include gorilla [7], cow [8], rabbit [9], and pig [10]. However, gaps in knowledge on the epidemiology, infectivity and pathogenicity of $S$. argenteus in humans and non-humans remains to be clarified. In the Netherlands, human cases have been described [3]. Here, we describe the first reported canine $S$. argenteus.

\section{Case}

A 9-year-old female Labrador retriever underwent a Tibia Plateau Levelling Osteotomy (TPLO) procedure after cranial cruciate ligament rupture in October 2019. She had a wound infection after 6 days under oral amoxicillin/clavulanic acid (12.5 mg/kg) prophylaxis, appearing superficial at first with increasing wound exudate and subsequent purulent drainage, without systemic symptoms. The wound infection was then empirically treated 
with oral doxycycline $(10 \mathrm{mg} / \mathrm{kg}$ ) for 10 days with success, allowing the wound to close. However, the wound reopened spontaneously 13 days after antibiotic treatment cessation and bacterial cultures were taken, after which targeted oral enrofloxacin $(5 \mathrm{mg} / \mathrm{kg})$ was prescribed for 14 days after positive cultures for Corynebacterium auris. Three weeks later, the wound infection persisted and a deep infection was suspected. Arthrocentesis was performed, with peroperative deep bacterial cultures yielding both Pseudomonas aeruginosa and Staphylococcus argenteus. The veterinary clinic decided not to treat the enrofloxacinsensitive Pseudomonas aeruginosa. Oral doxycycline $(10 \mathrm{mg} / \mathrm{kg})$ was reinitiated to treat the $S$. argenteus infection, for another 3 weeks, with treatment success.

\section{Materials and Methods}

Bacterial cultures were performed on sheep blood agar plates (bioTRADING Benelux B.V., Mijdrecht, the Netherlands). Staphylococcus spp. was suspected based on colony morphology in routine diagnostics. The $S$. argenteus colony morphology appeared as creamy white 'argent' colonies with a $\beta$-hemolytic zone (Figure 1). Overnight colony material was smeared on an MSP 96 target, and 1 uL of 70\% formic acid was added, followed by addition of $1 \mathrm{uL}$ of MALDI matrix solution (Bruker Corporation, Billerica, MA, USA). The MALDI-TOF MS (Bruker Corporation, Billerica, MA, USA) analyses were performed in duplicate and resulted in the identification of S. argenteus with scores of 2.19 and 2.26 .

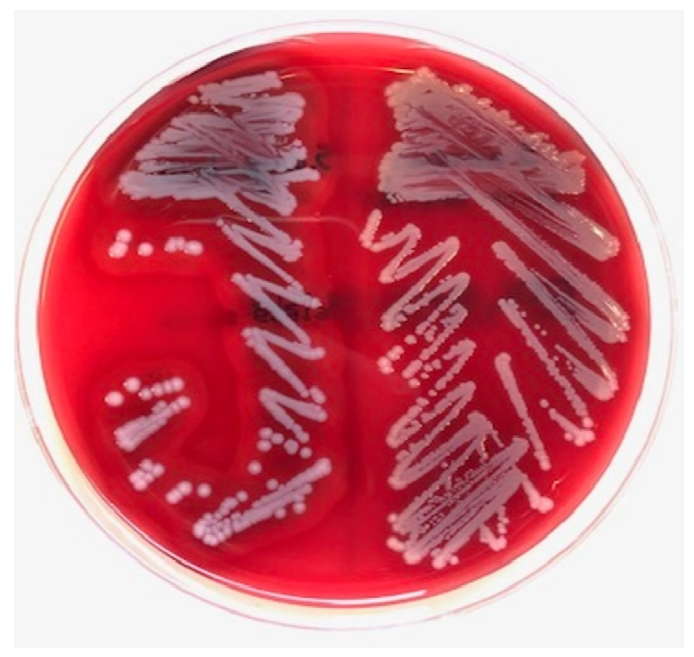

Figure 1. Colony morphology of found Staphylococcus argenteus (left), showing creamy white colonies with a $\beta$-hemolytic zone on sheep blood agar. Staphylococcus aureus (right) for comparison.

DNA was isolated using the DNeasy Ultra Clean Microbial kit (Qiagen, Venlo, The Netherlands) and was not sheared and size-selected for sequencing. For whole-genome sequencing, an Illumina library was prepared using the Nextera kit and pooled libraries were sequenced using Nextseq 500 (Illumina, San Diego, CA, USA). Nanopore long-read sequencing was performed according to protocol (SQK-LSK109) on a MinION device (Oxford Nanopore Technologies, Oxford Science Park, UK). Hybrid assembly was performed using Unicycler v0.4.9b [11], and the reads and complete assembled genome of isolate 20S00001-1 are available from the European Nucleotide Archive browser at http: / / www.ebi.ac.uk/ena/browser/view / GCA_927312875 (accessed on 25 January 2022). Using in silico multilocus sequence typing (MLST) [12], the strain was identified to belong to sequence type ST2250. Using AMRFinderPlus [13], two likely chromosomal resistance genes were identified; tet(38) and fosB. For phylogenetic analysis (Figure 2), a tree based on singlenucleotide polymorphisms (SNPs) in the core genome was constructed using the genome alignment made with parsnp v1.2 [14] with filtering of recombination regions using gubbins v 2.3.4. [15]. All available S. argenteus and S. schweitzeri isolates from the MLST database 
(accessed on 13 July 2021, https:/ / pubmlst.org/organisms/staphylococcus-aureus/) were included. Publicly available $S$. aureus reference isolates of the most common S. aureus clonal complexes, sequence types ST5, ST8, ST22, ST30 and ST45, were used for rooting [16].

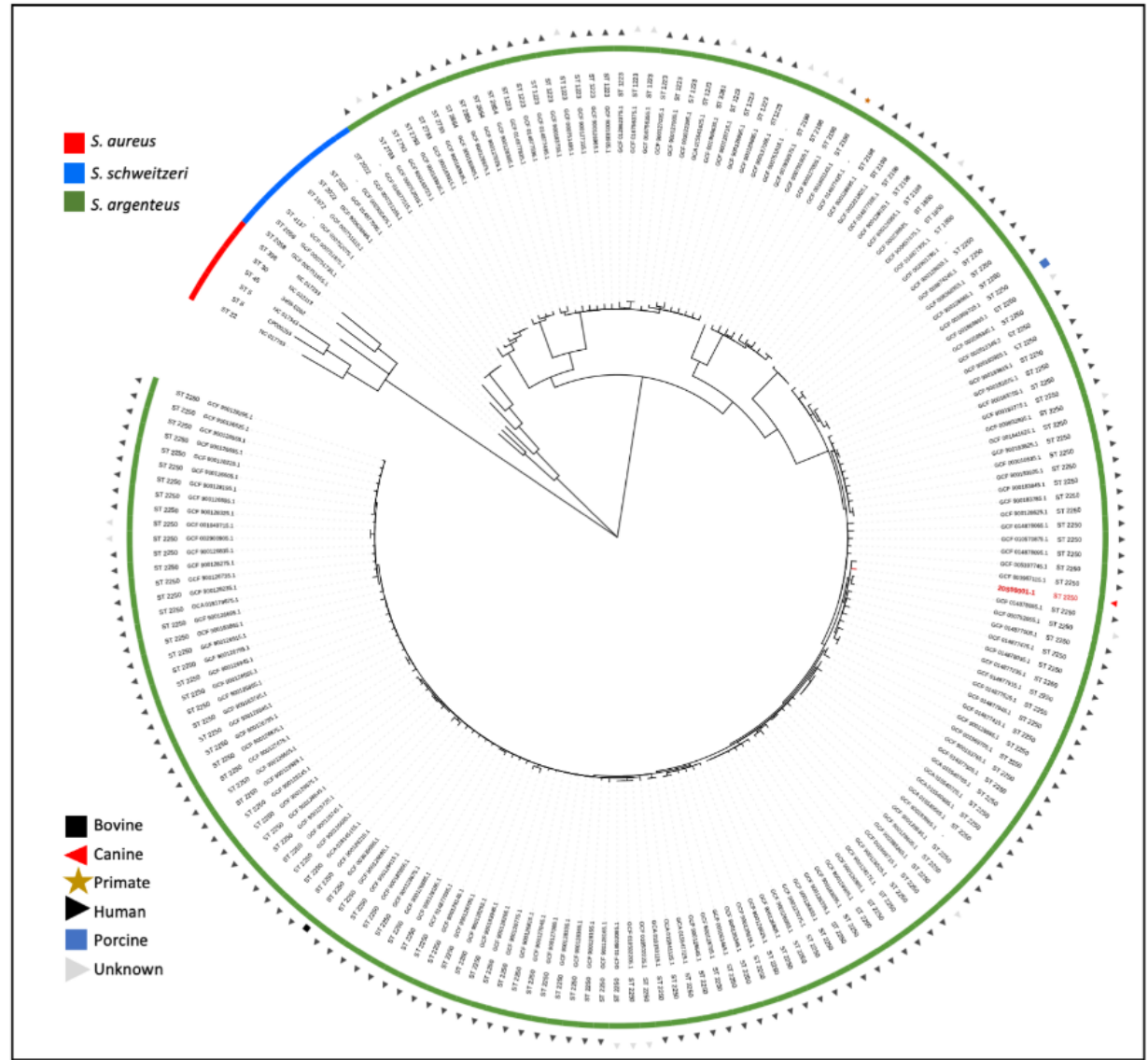

Figure 2. Phylogenetic tree of the core genome SNPs diversity of Staphylococcus argenteus, Staphylococcus schweitzeri and Staphylococcus aureus. The identified S. argenteus ST2250 isolate 20S00001-1 is indicated in red. Branch lengths in the tree were adjusted to sites per core genome and square root transformed to enhance resolution. All publicly available S. argenteus and S. schweitzeri isolates were included. Available S. aureus reference isolates of the most common S. aureus clonal complexes were used for rooting.

Using the virulence factor database [17] for this isolate, genes were found related to adherence virulence factors including autolysins (atl), adhesins (ebh, ebp, eap, efb, fnbA/B, $s d r C-H$ and $s p a)$ and adhesins critical for biofilm formation $(i c a A-C, R)$. Staphylocoagulase (coa) and thermonuclease (nuc1) were identified. Genes related to immune evasion were $s c n$ and $s b i$, as well as several genes responsible for exoenzyme and exotoxin secretion with the Type VII secretion system (esaA,B; esaG; ess $A-C$ and es $x A-D)$ and multiple undetermined capsule genes. Toxins included hemolysins ( $h l a, h l d, h \lg A-C)$, multiple undetermined exotoxins, staphylococcal enterotoxins and enterotoxin-like toxins (sec, selq), and leukocidins $(l u k D)$. Panton-Valentine leucocidin $(l u k F / S)$ and toxic shock syndrome toxin $(t s t / t s s t)$ were negative.

For phenotypic detection of resistance, a combination of agar diffusion with automated antibiogram (BD Phoenix system; Becton, Dickinson and Company, Franklin Lakes, NJ, USA) and microdilution (custom-made microdilution plates; MERLIN Diagnostika $\mathrm{GmbH}$, Bornheim, Germany) were used for human and veterinary antibiotics, respectively. Fosfomycin MIC was verified by Etest (BioMérieux, Marcy l'Etoile, France). Antibiogram methods used are common practice and used in clinical diagnostics. EUCAST (human) [18] 
and CLSI (veterinary) [19] clinical breakpoints were applied; results are listed in Table 1. Phenotypic antibiotic resistance was identified for fosfomycin.

Table 1. Phenotypic detection of resistance. A combination of agar diffusion with automated antibiogram (BD Phoenix system; Becton, Dickinson and Company, Franklin Lakes, NJ, USA) and microdilution (custom-made microdilution plates; MERLIN Diagnostika GmbH, Bornheim, Germany) were used. Fosfomycin MIC was verified by Etest (BioMérieux, Marcy l'Etoile, France). ${ }^{\S}$ EUCAST clinical breakpoints were applied, http:/ / www.eucast.org (accessed on 25 January 2022) [18]. ₹ CLSI breakpoints were applied for bacteria isolated from animals, https:/ /clsi.org (accessed on 25 January 2022) [19].

\begin{tabular}{|c|c|c|}
\hline Antibiotic & MIC or Disk Zone & Interpretation \\
\hline \multicolumn{3}{|c|}{ Agar diffusion (mm) } \\
\hline Cefoxitin & 29 & Susceptible $\S$ \\
\hline Clindamycin & 28 & Susceptible $\S$ \\
\hline Rifampicin & 32 & Susceptible $\S$ \\
\hline Trimethoprim & 26 & Susceptible $\S$ \\
\hline \multicolumn{3}{|c|}{ Automated antibiogram $(\mathrm{mg} / \mathrm{L})$} \\
\hline Ceftaroline & 0.5 & Susceptible $\S$ \\
\hline Chloramphenicol & 8 & Susceptible $\S$ \\
\hline Ciprofloxacin & $\leq 0.5$ & Susceptible $\S$ \\
\hline Daptomycin & 0.5 & Susceptible $\S$ \\
\hline Erythromycin & $\leq 0.2$ & Susceptible $\S$ \\
\hline Fusidic acid & $\leq 0.5$ & Susceptible $\S$ \\
\hline Gentamycin & $\leq 1$ & Susceptible $\S$ \\
\hline Levofloxacin & $\leq 0.5$ & Susceptible $\S$ \\
\hline Linezolid & 2 & Susceptible $\S$ \\
\hline Moxifloxacin & $\leq 0.25$ & Susceptible $\S$ \\
\hline Mupirocin & $\leq 0.5$ & Susceptible $\S$ \\
\hline Penicillin & 0.125 & Susceptible $\S$ \\
\hline Quinupristine/Dalfopristine & $\leq 0.5$ & Susceptible $\S$ \\
\hline Teicoplanin & $\leq 0.5$ & Susceptible $\S$ \\
\hline Tetracycline & $\leq 0.5$ & Susceptible $\S$ \\
\hline Tigecycline & $\leq 0.125$ & Susceptible $\S$ \\
\hline Tobramycin & $\leq 1$ & Susceptible $\S$ \\
\hline Trimethoprim/sulfamethoxazole & $\leq 0.5$ & Susceptible $\S$ \\
\hline Vancomycin & 1 & Susceptible $\S$ \\
\hline \multicolumn{3}{|c|}{ Microdilution (mg/L) } \\
\hline Enrofloxacin & $\leq 0.25$ & Susceptible $\ddagger$ \\
\hline Kanamycin & $\leq 16$ & Susceptible ${ }^{\ddagger}$ \\
\hline Neomycin & $\leq 8$ & Susceptible $\ddagger$ \\
\hline \multicolumn{3}{|c|}{ Epsilometer test (mg/L) } \\
\hline Fosfomycin & 64 & Resistant $\S$ \\
\hline
\end{tabular}




\section{Discussion}

Here, we describe the first canine $S$. argenteus reported to date. Colony morphology, coagulase testing and $16 \mathrm{~S}$ rRNA sequencing cannot distinguish S. argenteus from S. aureus, but an updated MALDI-TOF MS database can reliably identify $S$. argenteus profiles that are present in the database [20]. MALDI-TOF MS and whole-genome sequencing are currently regarded as valid diagnostic tools [2], while only the latter can provide definite identification [1]. For routine identification of the canine isolate, MALDI-TOF MS and subsequent whole-genome sequencing were employed. The reported canine S. argenteus isolate genetically belongs to the most frequent isolated lineage, ST2250 [5].

Because of its recent discovery [1] and limited known genetical subtypes, it is currently unclear whether S. argenteus has been endemic worldwide or recently spread globally [5]. This will be a challenge to address with the current problematic delimitation of $S$. argenteus from S. aureus in routine diagnostics and the relatively high costs of whole-genome sequencing. There are also no known clinical consequences of misidentifying $S$. argenteus as $S$. aureus due to their seemingly comparable pathogenicity. In the Netherlands, multiple methicillin-resistant $S$. argenteus have been identified retrospectively from human clinical and outpatient settings [3]. Two patients had likely imported the isolates from hospitals in Australia and Philippines-known geographical 'hot spots' of S. argenteus- [2] —-while others had an unknown source. Regarding methicillin resistance in S. argenteus, routine phenotypic and genotypic approaches for S. aureus are applicable, and infection prevention and control measures are equal.

S. argenteus has been reported to harbor a multitude of virulence factors common in well-characterized S. aureus lineages $[2,5,21]$, associated with $S$. aureus pathogenicity and causing a similar spectrum of disease. Comparing virulence genes identified in the canine isolate with neighboring human isolates within the phylogenetic tree, no unique genes were identified, thus no genetic explanation could be found for its interaction with a canine host. In fact, this isolate had fewer virulence genes and toxins when compared with the isolates found in humans, comparable to the isolate found in a gorilla [7]. Interestingly, from all genomes shown in Figure 2, two closely related genomes (GCF_003967115.1 and GCF_014877235.1) harbored the tsst-1 gene coding for toxic shock syndrome toxin1. Panton-Valentine leucocidin $(l u k F / S)$ and toxic shock syndrome toxin $(t s t / t s s t)$ were, however, not identified in this isolate.

The fos $B$ and tet(38) genes identified in this S. argenteus isolate did phenotypically confer resistance to fosfomycin but not tetracycline (Table 1). Doxycycline resulted in treatment success, as was expected with proven tetracycline susceptibility. Tet38 is a known chromosomally encoded native efflux pump in S. aureus, which can confer resistance if (additionally) plasmid encoded [22]. The fos $B$ gene also does not always confer phenotypic resistance $[23,24]$ and is postulated to influence cellular processes, including adaptation to reactive oxygen species [25], while the drug fosfomycin is also known to be naturally produced by Streptomyces spp. [26] and Pseudomonas spp. [27]. An environmental origin can therefore not be excluded. However, fosfomycin is a drug commonly used in human medicine and $f_{S} B$ resistance genes have been commonly reported in S. aureus clinical methicillin-resistant $S$. aureus (MRSA) strains, although rare in methicillin-susceptible $S$. aureus (MSSA) [28]. Recently, fosB resistance genes were found in canine methicillin-resistant Staphylococcus pseudintermedius (MRSP) isolates [24] and in several coagulase-negative staphylococci in colony-born and wild vervet monkeys on the small island of Saint Kitts [29]. Fosfomycin is rarely used in veterinary medicine, which raises questions regarding the source of the fos $B$ gene in animal isolates. The presence of fos $B$ hypothetically might benefit survival due to its effects on cellular processes, rather than antibiotic resistance. Interestingly, in a recent genomic analysis of 132 known global S. argenteus strains, about $90 \%$ of the isolates were found to carry highly similar, likely chromosomally encoded fos $B$ genes [23]. Taken together, fos $B$ is commonly found in S. argenteus, but we cannot confer environmental, human or non-human origin. 
The S. argenteus ST2198 and ST2250 found in animals are abundantly found in humans (Figure 2). The relative lack of virulence factors and antibiotic resistance in the found canine isolate, whilst causing disease early postoperatively, underscores its intrinsic pathogenicity. As doxycycline resulted in treatment success, this argues in favor of the clinical relevance of $S$. argenteus in this patient. It was unclear how the dog acquired the isolate, but the lack of travel history suggests this strain was acquired locally. Foodborne transmission has been described for S. argenteus [30], but the dog did not receive raw foods. Unfortunately, due to loss of follow-up, no swabs were taken from household members to evaluate $S$. argenteus colonization. Specific history from household members could not be taken, but they did not report any skin and soft tissue infections at the time of veterinary visit.

\section{Conclusions}

We report the first canine $S$. argenteus isolate, undoubtedly locally acquired, belonging to the most frequent isolated lineage ST2250. Using whole-genome sequencing, we found clear overlap in virulence genes with $S$. aureus. Resistance to fosfomycin was identified both phenotypically and genetically, but no genetic explanation could be found for its interaction with a canine host. Our finding underscores its multispecies pathogenicity and its potential (anthropo-)zoonotic spread.

Author Contributions: Conceptualization, E.F.J.M., L.v.d.G.-v.B., B.D., E.M.B.; Methodology, E.F.J.M., A.v.R., I.M., L.v.d.G.-v.B., B.D., E.M.B.; Software, L.v.d.G.-v.B., B.D.; Formal Analysis, E.F.J.M., L.v.d.G.v.B., B.D., E.M.B.; Investigation, A.v.R., E.M.B.; Resources, E.M.B., I.M.; Data Curation, E.F.J.M., L.v.d.G.-v.B., B.D.; Writing-Original Draft Preparation, E.F.J.M.; Writing-Review and Editing, E.F.J.M., A.v.R., I.M., L.v.d.G.-v.B., B.D., E.M.B.; Visualization, L.v.d.G.-v.B., B.D., E.M.B.; Supervision, I.M., E.M.B. All authors have read and agreed to the published version of the manuscript.

Funding: This research received no external funding.

Institutional Review Board Statement: Ethical review and approval were waived, due to the observational and non-experimental nature of the study.

Informed Consent Statement: Not applicable.

Data Availability Statement: The reads and complete assembled genome of S. argenteus isolate 20S00001-1 are available from the European Nucleotide Archive browser at http:/ /www.ebi.ac.uk/ ena/browser/view/GCA_927312875 accessed on 25 Januanry 2022.

Conflicts of Interest: The authors declare no conflict of interest.

\section{References}

1. Tong, S.Y.C.; Schaumburg, F.; Ellington, M.J.; Corander, J.; Pichon, B.; Leendertz, F.; Bentley, S.D.; Parkhill, J.; Holt, D.; Peters, G.; et al. Novel staphylococcal species that form part of a Staphylococcus aureus-related complex: The non-pigmented Staphylococcus argenteus sp. nov. and the non-human primate-associated Staphylococcus schweitzeri sp. nov. Int. J. Syst. Evol. Microbiol. 2015, 65 Pt 1, 15-22. [CrossRef] [PubMed]

2. Becker, K.; Schaumburg, F.; Kearns, A.; Larsen, A.; Lindsay, J.; Skov, R.; Westh, H. Implications of identifying the recently defined members of the Staphylococcus aureus complex S. argenteus and S. schweitzeri: A position paper of members of the ESCMID Study Group for Staphylococci and Staphylococcal Diseases (ESGS). Clin. Microbiol. Infect. 2019, 25, 1064-1070. [CrossRef] [PubMed]

3. Bank, L.E.A.; Bosch, T.; Schouls, L.M.; Weersink, A.J.L.; Witteveen, S.; Wolffs, P.F.G.; Nijhuis, R.H.T. Methicillin-resistant Staphylococcus argenteus in the Netherlands: Not a new arrival. Eur. J. Clin. Microbiol. Infect. Dis. 2021, 40, 1583-1585. [CrossRef] [PubMed]

4. Grossmann, A.; Froböse, N.J.; Mellmann, A.; Alabi, A.S.; Schaumburg, F.; Niemann, S. An in vitro study on Staphylococcus schweitzeri virulence. Sci. Rep. 2021, 11, 1157. [CrossRef]

5. Zhang, D.-F.; Zhi, X.-Y.; Zhang, J.; Paoli, G.C.; Cui, Y.; Shi, C.; Shi, X. Preliminary comparative genomics revealed pathogenic potential and international spread of Staphylococcus argenteus. BMC Genom. 2017, 18, 808. [CrossRef]

6. Thaipadungpanit, J.; Amornchai, P.; Nickerson, E.K.; Wongsuvan, G.; Wuthiekanun, V.; Limmathurotsakul, D.; Peacock, S.J. Clinical and Molecular Epidemiology of Staphylococcus argenteus Infections in Thailand. J. Clin. Microbiol. 2015, 53, 1005. [CrossRef]

7. Schuster, D.; Rickmeyer, J.; Gajdiss, M.; Thye, T.; Lorenzen, S.; Reif, M.; Josten, M.; Szekat, C.; Melo, L.D.; Schmithausen, R.M.; et al. Differentiation of Staphylococcus argenteus (formerly: Staphylococcus aureus clonal complex 75) by mass spectrometry from S. aureus using the first strain isolated from a wild African great ape. Int. J. Med Microbiol. 2017, 307, 57-63. [CrossRef] 
8. Pumipuntu, N.; Tunyong, W.; Chantratita, N.; Diraphat, P.; Pumirat, P.; Sookrung, N.; Chaicumpa, W.; Indrawattana, N. Staphylococcus spp. associated with subclinical bovine mastitis in central and northeast provinces of Thailand. PeerJ 2019, 7, e6587. [CrossRef]

9. Indrawattana, N.; Pumipuntu, N.; Suriyakhun, N.; Jangsangthong, A.; Kulpeanprasit, S.; Chantratita, N.; Sookrung, N.; Chaicumpa, W.; Buranasinsup, S. Staphylococcus argenteusfrom rabbits in Thailand. Microbiologyopen 2018, 8, e00665. [CrossRef]

10. Kaden, R.; Engstrand, L.; Rautelin, H.; Johansson, C. Which methods are appropriate for the detection of Staphylococcus argenteus and is it worthwhile to distinguish S. argenteus from S. aureus? Infect. Drug Resist. 2018, 11, 2335-2344. [CrossRef]

11. Wick, R.R.; Judd, L.M.; Gorrie, C.L.; Holt, K.E. Unicycler: Resolving bacterial genome assemblies from short and long sequencing reads. PLoS Comput. Biol. 2017, 13, e1005595. [CrossRef] [PubMed]

12. Larsen, M.V.; Cosentino, S.; Rasmussen, S.; Friis, C.; Hasman, H.; Marvig, R.L.; Jelsbak, L.; Sicheritz-Ponten, T.; Ussery, D.W.; Aarestrup, F.M.; et al. Multilocus Sequence Typing of Total-Genome-Sequenced Bacteria. J. Clin. Microbiol. 2012, 50, 1355-1361. [CrossRef] [PubMed]

13. Feldgarden, M.; Brover, V.; Haft, D.H.; Prasad, A.B.; Slotta, D.J.; Tolstoy, I.; Tyson, G.H.; Zhao, S.; Hsu, C.; McDermott, P.F.; et al. Validating the AMRFinder Tool and Resistance Gene Database by Using Antimicrobial Resistance Geno-type-Phenotype Correlations in a Collection of Isolates. Antimicrob. Agents Chemother. 2019, 63, e00483-19. [CrossRef] [PubMed]

14. Treangen, T.J.; Ondov, B.D.; Koren, S.; Phillippy, A.M. The Harvest suite for rapid core-genome alignment and visualization of thousands of intraspecific microbial genomes. Genome Biol. 2014, 15, 524. [CrossRef]

15. Croucher, N.J.; Page, A.J.; Connor, T.R.; Delaney, A.J.; Keane, J.A.; Bentley, S.D.; Parkhill, J.; Harris, S.R. Rapid phylogenetic analysis of large samples of recombinant bacterial whole genome sequences using Gubbins. Nucleic Acids Res. 2014, 43, e15. [CrossRef]

16. Jolley, K.A.; Bray, J.E.; Maiden, M.C.J. Open-access bacterial population genomics: BIGSdb software, the PubMLST.org website and their applications. Wellcome Open Res. 2018, 3, 124. [CrossRef]

17. Liu, B.; Zheng, D.; Jin, Q.; Chen, L.; Yang, J. VFDB 2019: A comparative pathogenomic platform with an interactive web interface. Nucleic Acids Res. 2019, 47, D687-D692. [CrossRef]

18. The European Committee on Antimicrobial Susceptibility Testing. Breakpoint Tables for Interpretation of MICs and Zone Diameters. Version 11.0. 2021. Available online: http: / / www.eucast.org (accessed on 25 January 2022).

19. CLSI. Clinical and Laboratory Standards Institute: Performance Standards for Antimicrobial Disk and Dilution Susceptibility Tests for Bacteria Isolated from Animals, 5th ed.; CLSI Supplement VET01S; Clinical and Laboratory Standards Institute: Malvern, PA, USA, 2020.

20. Chen, S.-Y.; Lee, H.; Teng, S.-H.; Wang, X.-M.; Lee, T.-F.; Huang, Y.-C.; Liao, C.-H.; Teng, L.-J.; Hsueh, P.-R. Accurate differentiation of novel Staphylococcus argenteus from Staphylococcus aureus using MALDI-TOF MS. Future Microbiol. 2018, 13, 997-1006. [CrossRef]

21. Jones, D.; Meijer, E.F.J.; Blatter, C.; Liao, S.; Pereira, E.R.; Bouta, E.M.; Jung, K.; Chin, S.M.; Huang, P.; Munn, L.L.; et al. Methicillinresistant Staphylococcus aureus causes sustained collecting lymphatic vessel dysfunction. Sci. Transl. Med. 2018, 10, eaam7964. [CrossRef]

22. Chen, C.; Hooper, D.C. Effect of Staphylococcus aureus Tet38 native efflux pump on in vivo response to tetracycline in a murine subcutaneous abscess model. J. Antimicrob. Chemother. 2017, 73, 720-723. [CrossRef]

23. Goswami, C.; Fox, S.; Holden, M.; Leanord, A.; Evans, T.J. Genomic Analysis of Global Staphylococcus argenteus Strains Reveals Distinct Lineages With Differing Virulence and Antibiotic Resistance Gene Content. Front. Microbiol. 2021, 12, 795173. [CrossRef] [PubMed]

24. DiCicco, M.; Weese, S.; Neethirajan, S.; Rousseau, J.; Singh, A. Fosfomycin susceptibility of canine methicillin-resistant Staphylococcus pseudintermedius isolates. Res. Vet. Sci. 2014, 96, 251-253. [CrossRef] [PubMed]

25. Helmann, J.D. Bacillithiol, a New Player in Bacterial Redox Homeostasis. Antioxid. Redox Signal. 2011, 15, 123-133. [CrossRef] [PubMed]

26. Hendlin, D.; Stapley, E.O.; Jackson, M.; Wallick, H.; Miller, A.K.; Wolf, F.J.; Miller, T.W.; Chaiet, L.; Kahan, F.M.; Foltz, E.L.; et al. Phosphonomycin, a New Antibiotic Produced by Strains of Streptomyces. Science 1969, 166, 122-123. [CrossRef]

27. Woodyer, R.D.; Shao, Z.; Thomas, P.M.; Kelleher, N.L.; Blodgett, J.A.; Metcalf, W.W.; van der Donk, W.A.; Zhao, H. Heterologous Production of Fosfomycin and Identification of the Minimal Biosynthetic Gene Cluster. Chem. Biol. 2006, 13, 1171-1182. [CrossRef]

28. Lee, Y.-C.; Chen, P.-Y.; Wang, J.-T.; Chang, S.-C. Prevalence of fosfomycin resistance and gene mutations in clinical isolates of methicillin-resistant Staphylococcus aureus. Antimicrob. Resist. Infect. Control 2020, 9, 135. [CrossRef]

29. Hoefer, A.; Boyen, F.; Beierschmitt, A.; Moodley, A.; Roberts, M.; Butaye, P. Methicillin-Resistant and Methicillin-Susceptible Staphylococcus from Vervet Monkeys (Chlorocebus sabaeus) in Saint Kitts. Antibiotics 2021, 10, 290. [CrossRef]

30. Wakabayashi, Y.; Takemoto, K.; Iwasaki, S.; Yajima, T.; Kido, A.; Yamauchi, A.; Kuroiwa, K.; Kumai, Y.; Yoshihara, S.; Tokumoto, H.; et al. Isolation and characterization of Staphylococcus argenteus strains from retail foods and slaughterhouses in Japan. Int. J. Food Microbiol. 2021, 363, 109503. [CrossRef] 